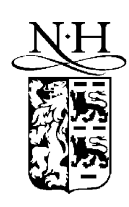

ELSEVIER

\title{
Application of Legendre-Bernstein basis transformations to degree elevation and degree reduction
}

\author{
Byung-Gook Lee ${ }^{\mathrm{a}, *}$, Yunbeom Park ${ }^{\mathrm{b}}$, Jaechil Yoo $^{\mathrm{c}}$ \\ a Division of Internet Engineering, Dongseo University, Busan, 617-716, South Korea \\ $\mathrm{b}$ Department of Mathematics Education, Seowon University, Cheongju, 361-742, South Korea \\ c Department of Mathematics, Dongeui University, Busan, 614-714, South Korea
}

Received 29 July 2002; received in revised form 29 July 2002

\begin{abstract}
We study the relationship of transformations between Legendre and Bernstein basis. Using the relationship, we present a simple and efficient method for optimal multiple degree reductions of Bézier curves with respect to the $L_{2}$-norm.

() 2002 Elsevier Science B.V. All rights reserved.
\end{abstract}

Keywords: Legendre; Bernstein; Basis transformations; Bézier curves; Degree elevation; Degree reduction

\section{Introduction}

We can express a polynomial curve with an appropriate basis for its use. The use of orthogonal basis such as Chebyshev and Legendre polynomial permits optimal degree reduction to exchange, convert or reduce data, or compare geometric entities which is an important task in CAGD (Li and Zhang, 1998; Mazure, 1999). For example, we have seen the use of Chebyshev and Legendre polynomial in degree reduction schemes (Watkins and Worsey, 1988; Eck, 1993, 1995). On the other hand, the Bernstein form of a polynomial having the recursive formula and the property of partition of unity offers valuable insight into its geometrical behavior, and has won widespread acceptance as the basis for Bézier curves and surfaces in CAGD (Farin, 1993). But Bernstein polynomials are not orthogonal. So the basis transformation is important and has been studied in many ways. Farouki (2000) found the explicit form of the basis transformation between Legendre and Bernstein basis.

\footnotetext{
* Corresponding author.

E-mail address: lbg@dongseo.ac.kr (B.-G. Lee).
} 
In this paper, we find the relationships between the Gram matrix $Q_{n}$ and the Legendre-Bernstein basis transformation matrix $M_{n}$, the $M_{n}$ and the Bernstein-Legendre basis transformation matrix $M_{n}^{-1}$, and the orthogonal matrix $U_{n}$ and $M_{n}$. We also obtain the relationships between the basis transformation matrices $M_{n}, M_{n}^{-1}$ and the degree elevation matrix $T_{n}$, the basis transformation matrices and the degree reduction matrix.

This paper is organized as follows. We explain the degree $n$ Legendre and Bernstein basis, and their transformations in Section 2. We discuss the relationship among transformations, $M_{n}, M_{n}^{-1}$, eigenvalues of Gram matrix and an orthogonal matrix $U_{n}$ in Section 3. We present the explicit method to degree elevation and degree reduction of Bézier curves in Sections 4 and 5.

\section{Legendre and Bernstein basis}

The Bézier representation uses Bernstein polynomials as basis functions for the linear space of polynomials. In terms of the Bernstein polynomials of degree $n$,

$$
B_{i}^{n}(t)=\left(\begin{array}{l}
n \\
i
\end{array}\right)(1-t)^{n-i} t^{i}, \quad i=0,1, \ldots, n,
$$

a parametric polynomial curve $P_{n}(t)$ of degree $n(n>0)$ in the plane can be expressed as

$$
P_{n}(t)=\sum_{i=0}^{n} c_{i} B_{i}^{n}(t), \quad 0 \leqslant t \leqslant 1,
$$

where the $\left\{c_{i}\right\}_{i=0}^{n}$ are the set of $(n+1)$ control points.

The product of Bernstein polynomials is

$$
B_{i}^{n}(t) B_{j}^{m}(t)=\frac{\left(\begin{array}{c}
n \\
i
\end{array}\right)\left(\begin{array}{c}
m \\
j
\end{array}\right)}{\left(\begin{array}{c}
n+m \\
i+j
\end{array}\right)} B_{i+j}^{n+m}(t)
$$

and the integration is

$$
\int_{0}^{1} B_{k}^{n}(t) \mathrm{d} t=\frac{1}{n+1} .
$$

The Legendre polynomials constitute an orthonormal basis that is well suited to least-squares approximation. To emphasize symmetry properties they are traditionally defined on the interval $[-1,+1]$, but for our purpose it is preferable to map this to $[0,1]$. The Legendre polynomials $L_{n}(t)$ on $t \in[0,1]$ can be generated by the explicit form

$$
L_{n}(t)=\sqrt{2 n+1} \sum_{i=0}^{\lfloor n / 2\rfloor}\left(\begin{array}{c}
n \\
i, i
\end{array}\right)\left(t^{2}-t\right)^{i}(2 t-1)^{n-2 i},
$$

where $\left(\begin{array}{c}n \\ i, j\end{array}\right)=\frac{n !}{i ! j !(n-i-j) !}$. This gives, in the first few instances,

$$
\begin{aligned}
& L_{0}(t)=1, \\
& L_{1}(t)=\sqrt{3}(2 t-1),
\end{aligned}
$$




$$
\begin{aligned}
& L_{2}(t)=\sqrt{5}\left(6 t^{2}-6 t+1\right), \\
& L_{3}(t)=\sqrt{7}\left(20 t^{3}-30 t^{2}+12 t-1\right) .
\end{aligned}
$$

The orthonormality of these polynomials is expressed by the relation

$$
\int_{0}^{1} L_{j}(t) L_{k}(t) \mathrm{d} t= \begin{cases}1 & \text { if } j=k \\ 0 & \text { if } j \neq k\end{cases}
$$

Consider a polynomial $P_{n}(t)$ of degree $n$, expressed in the degree $n$ Bernstein and Legendre basis on $t \in[0,1]$ :

$$
P_{n}(t)=\sum_{j=0}^{n} c_{j} B_{j}^{n}(t)=\sum_{k=0}^{n} l_{k} L_{k}(t) .
$$

We are interested in the linear transformation

$$
c_{j}=\sum_{k=0}^{n} M_{n}(j, k) l_{k}, \quad j=0,1, \ldots, n,
$$

that maps the Legendre coefficients $l_{0}, l_{1}, \ldots, l_{n}$ into the Bernstein coefficients $c_{0}, c_{1}, \ldots, c_{n}$, and its inverse. Writing $c=\left[c_{0}, c_{1}, \ldots, c_{n}\right]^{\mathrm{t}}$ and $l=\left[l_{0}, l_{1}, \ldots, l_{n}\right]^{\mathrm{t}}$, we may express this in vector-matrix form as

$$
c=M_{n} l .
$$

Then we have the following theorem, see (Farouki, 2000).

Theorem 1. The Legendre polynomial $L_{k}(t)$ can be expressed in the Bernstein basis $B_{0}^{n}(t), B_{1}^{n}(t), \ldots$, $B_{n}^{n}(t)$ of degree $n$ as

$$
\begin{aligned}
L_{k}(t) & =\sqrt{2 k+1} \sum_{i=0}^{k}(-1)^{k+i}\left(\begin{array}{l}
k \\
i
\end{array}\right) B_{i}^{k}(t) \\
& =\sum_{j=0}^{n} \frac{\sqrt{2 k+1}}{\left(\begin{array}{l}
n \\
j
\end{array}\right)} \sum_{i=\max (0, j+k-n)}^{\min (j, k)}(-1)^{k+i}\left(\begin{array}{l}
k \\
i
\end{array}\right)\left(\begin{array}{c}
k \\
i
\end{array}\right)\left(\begin{array}{c}
n-k \\
j-i
\end{array}\right) B_{j}^{n}(t) .
\end{aligned}
$$

The elements of the matrix $M_{n}$ that transforms the Legendre coefficients of degree $n$ polynomials into the Bernstein coefficients according to equation, are given for $0 \leqslant j, k \leqslant n$ by

$$
M_{n}(j, k)=\frac{\sqrt{2 k+1}}{\left(\begin{array}{l}
n \\
j
\end{array}\right)} \sum_{i=\max (0, j+k-n)}^{\min (j, k)}(-1)^{k+i}\left(\begin{array}{l}
k \\
i
\end{array}\right)\left(\begin{array}{l}
k \\
i
\end{array}\right)\left(\begin{array}{c}
n-k \\
j-i
\end{array}\right) .
$$

For Bernstein to Legendre transformation matrix $M_{n}^{-1}$, see (Farouki, 2000).

Theorem 2. The elements of the inverse $M_{n}^{-1}$ are given for $0 \leqslant j, k \leqslant n$ by

$$
M_{n}^{-1}(j, k)=\frac{\sqrt{2 j+1}}{n+j+1} \frac{1}{\left(\begin{array}{c}
n+j \\
n
\end{array}\right)} \sum_{i=0}^{j}(-1)^{j+i}\left(\begin{array}{c}
j \\
i
\end{array}\right)\left(\begin{array}{c}
k+i \\
k
\end{array}\right)\left(\begin{array}{c}
n-k+j-i \\
n-k
\end{array}\right) .
$$




\section{Example 1.}

$$
\begin{aligned}
& M_{1}=\left[\begin{array}{cc}
1 & -\sqrt{3} \\
1 & \sqrt{3}
\end{array}\right], \quad M_{1}^{-1}=\left[\begin{array}{cc}
\frac{1}{2} & \frac{1}{2} \\
-\frac{\sqrt{3}}{6} & \frac{\sqrt{3}}{6}
\end{array}\right], \\
& M_{2}=\left[\begin{array}{ccc}
1 & -\sqrt{3} & \sqrt{5} \\
1 & 0 & -2 \sqrt{5} \\
1 & \sqrt{3} & \sqrt{5}
\end{array}\right], \quad M_{2}^{-1}=\left[\begin{array}{ccc}
\frac{1}{3} & \frac{1}{3} & \frac{1}{3} \\
-\frac{\sqrt{3}}{6} & 0 & \frac{\sqrt{3}}{6} \\
\frac{\sqrt{5}}{30} & -\frac{2 \sqrt{5}}{30} & \frac{\sqrt{5}}{30}
\end{array}\right] \text {, } \\
& M_{3}=\left[\begin{array}{cccc}
1 & -\sqrt{3} & \sqrt{5} & -\sqrt{7} \\
1 & -\frac{\sqrt{3}}{3} & -\sqrt{5} & 3 \sqrt{7} \\
1 & \frac{\sqrt{3}}{3} & -\sqrt{5} & -3 \sqrt{7} \\
1 & \sqrt{3} & \sqrt{5} & \sqrt{7}
\end{array}\right], \quad M_{3}^{-1}=\left[\begin{array}{cccc}
\frac{1}{4} & \frac{1}{4} & \frac{1}{4} & \frac{1}{4} \\
-\frac{3 \sqrt{3}}{20} & -\frac{\sqrt{3}}{20} & \frac{\sqrt{3}}{20} & \frac{3 \sqrt{3}}{20} \\
\frac{\sqrt{5}}{20} & -\frac{\sqrt{5}}{20} & -\frac{\sqrt{5}}{20} & \frac{\sqrt{5}}{20} \\
-\frac{\sqrt{7}}{140} & \frac{3 \sqrt{7}}{140} & -\frac{3 \sqrt{7}}{140} & \frac{\sqrt{7}}{140}
\end{array}\right] \text {. }
\end{aligned}
$$

\section{3. $L_{2}$-norm of the polynomial $P_{n}$}

We compute the $L_{2}$-norm of a Bézier curve of degree $n$. From these equations (1) and (2), we obtain the following computation for the $L_{2}$-norm of the polynomial $P_{n}$ with Bernstein basis:

$$
\begin{aligned}
\left\|P_{n}\right\|_{2}^{2} & =\int_{0}^{1}\left|\sum_{i=0}^{n} c_{i} B_{i}^{n}(t)\right|^{2} \mathrm{~d} t=\int_{0}^{1} \sum_{i, j} c_{i} c_{j} B_{i}^{n}(t) B_{j}^{n}(t) \mathrm{d} t \\
& =\sum_{i, j} c_{i} c_{j} \int_{0}^{1} \frac{\left(\begin{array}{c}
n \\
i
\end{array}\right)\left(\begin{array}{c}
n \\
j
\end{array}\right)}{\left(\begin{array}{c}
2 n \\
i+j
\end{array}\right)} B_{i+j}^{2 n}(t) \mathrm{d} t=\frac{1}{2 n+1} \sum_{i, j} c_{i} c_{j} \frac{\left(\begin{array}{c}
n \\
i
\end{array}\right)\left(\begin{array}{c}
n \\
j
\end{array}\right)}{\left(\begin{array}{c}
2 n \\
i+j
\end{array}\right)} .
\end{aligned}
$$

Let the elements of the Gram matrix $Q_{n}$ of the Bernstein basis be the

$$
Q_{n}(i, j)=\frac{1}{2 n+1} \frac{\left(\begin{array}{c}
n \\
i
\end{array}\right)\left(\begin{array}{l}
n \\
j
\end{array}\right)}{\left(\begin{array}{c}
2 n \\
i+j
\end{array}\right)}, \quad i, j=0,1, \ldots, n .
$$

Then the $L_{2}$-norm of the polynomial $P_{n}$ is

$$
\left\|P_{n}\right\|_{2}^{2}=c^{\mathrm{t}} Q_{n} c .
$$

Here are some examples of $Q_{n}$.

\section{Example 2.}

$$
Q_{1}=\left[\begin{array}{cc}
\frac{1}{3} & \frac{1}{6} \\
\frac{1}{6} & \frac{1}{3}
\end{array}\right], \quad Q_{2}=\left[\begin{array}{ccc}
\frac{1}{5} & \frac{1}{10} & \frac{1}{30} \\
\frac{1}{10} & \frac{2}{15} & \frac{1}{10} \\
\frac{1}{30} & \frac{1}{10} & \frac{1}{5}
\end{array}\right], \quad Q_{3}=\left[\begin{array}{cccc}
\frac{1}{7} & \frac{1}{14} & \frac{1}{35} & \frac{1}{140} \\
\frac{1}{14} & \frac{3}{35} & \frac{9}{140} & \frac{1}{35} \\
\frac{1}{35} & \frac{9}{140} & \frac{3}{35} & \frac{1}{14} \\
\frac{1}{140} & \frac{1}{35} & \frac{1}{14} & \frac{1}{7}
\end{array}\right] .
$$


From the definition of the Gram matrix $Q_{n}$ and the mathematical induction, all the upper left submatrices of the Gram matrix $Q_{n}$ have positive determinants. So $Q_{n}$ is a real symmetric positive definite matrix, see (Lee and Park, 1997). Thus it can be diagonalized by an orthogonal matrix $U_{n}$ (i.e., $U_{n}^{-1}=U_{n}^{\mathrm{t}}$ ) whose column vectors are orthonormal eigenvectors of $Q_{n}$, that is,

$$
Q_{n}=U_{n} D_{n} U_{n}^{\mathrm{t}}
$$

where $D_{n}$ is the diagonal matrix with positive eigenvalues of the matrix $Q_{n}$.

The following theorem is the direct result from Proposition 10 in (Lyche and Scherer, 2000).

Theorem 3. From the Gram matrix $Q_{n}$ given by (6) we have

$$
Q_{n} M_{n}=M_{n} D_{n}
$$

where $\lambda_{k}=\frac{1}{2 n+1} \frac{\left(\begin{array}{c}2 n+1 \\ n+k\end{array}\right)}{\left(\begin{array}{c}2 n \\ n\end{array}\right)}(k=0,1, \ldots, n)$ are eigenvalues of the Gram matrix $Q_{n}$.

From the orthonormality (3) of Legendre basis, we obtain the following computation for the $L_{2}$-norm of the polynomial $P_{n}$ with Legendre basis:

$$
\left\|P_{n}\right\|_{2}{ }^{2}=\int_{0}^{1}\left|\sum_{i=0}^{n} l_{i} L_{i}(t)\right|^{2} \mathrm{~d} t=\sum_{i, j} l_{i} l_{j} \int_{0}^{1} L_{i}(t) L_{j}(t) \mathrm{d} t=l^{\mathrm{t}} l .
$$

From Theorem 3, we get the following theorem that describes the relationship among $M_{n}^{-1}, M_{n}$ and $D_{n}$.

Theorem 4. For the Bernstein to Legendre transformation matrix $M_{n}^{-1}$ we have

$$
M_{n}^{-1}=D_{n} M_{n}^{\mathrm{t}} .
$$

Proof. From (7) and (8), we have

$$
c^{\mathrm{t}} Q_{n} c=l^{\mathrm{t}} l .
$$

By the definition of $M_{n}^{-1}$, we can also express the $L_{2}$-norm of the polynomial $P_{n}$ as

$$
c^{\mathrm{t}} Q_{n} c=c^{\mathrm{t}}\left(M_{n}^{-1}\right)^{\mathrm{t}} M_{n}^{-1} c .
$$

By Theorem 3 and (9) we obtain

$$
Q_{n}=M_{n} D_{n} M_{n}^{-1}=\left(M_{n}^{-1}\right)^{\mathrm{t}} M_{n}^{-1} .
$$

Multiplying both sides by $M_{n}$ and considering the transpose of both sides, we complete the proof.

The following theorem enables us to compute $U_{n}$ with the explicit forms of $M_{n}$ and $D_{n}$.

Theorem 5. For the orthogonal matrix $U_{n}$ of the Gram matrix $Q_{n}$ we have

$$
U_{n}=M_{n}{\sqrt{D_{n}}}
$$


Proof. From Theorem 4, we have

$$
Q_{n}=M_{n} D_{n} M_{n}^{-1}=M_{n} D_{n} D_{n} M_{n}^{\mathrm{t}}=M_{n}{\sqrt{D_{n}}}_{n}\left(M_{n}{\sqrt{D_{n}}}^{\mathrm{t}} .\right.
$$

And we can easily check the orthogonality of $M_{n}{\sqrt{D_{n}}}_{n}$

$$
\left(M_{n}{\sqrt{D_{n}}}^{-1}={\sqrt{D_{n}}}^{-1} M_{n}^{-1}={\sqrt{D_{n}}}_{n}^{\mathrm{t}}=\left(M_{n}{\sqrt{D_{n}}}^{\mathrm{t}}{ }^{\mathrm{t}} .\right.\right.
$$

This completes the proof.

Here are some examples of $U_{n}$ and $D_{n}$.

\section{Example 3.}

$$
\begin{aligned}
U_{1} & =\left[\begin{array}{cc}
\frac{1}{\sqrt{2}} & -\frac{1}{\sqrt{2}} \\
\frac{1}{\sqrt{2}} & \frac{1}{\sqrt{2}}
\end{array}\right], \quad D_{1}=\left[\begin{array}{cc}
\frac{1}{2} & 0 \\
0 & \frac{1}{6}
\end{array}\right], \\
U_{2} & =\left[\begin{array}{ccc}
\frac{1}{\sqrt{3}} & -\frac{1}{\sqrt{2}} & \frac{1}{\sqrt{6}} \\
\frac{1}{\sqrt{3}} & 0 & -\frac{2}{\sqrt{6}} \\
\frac{1}{\sqrt{3}} & \frac{1}{\sqrt{2}} & \frac{1}{\sqrt{6}}
\end{array}\right], \quad D_{2}=\left[\begin{array}{ccc}
\frac{1}{3} & 0 & 0 \\
0 & \frac{1}{6} & 0 \\
0 & 0 & \frac{1}{30}
\end{array}\right], \\
U_{3} & =\left[\begin{array}{cccc}
\frac{1}{2} & -\frac{3}{\sqrt{20}} & \frac{1}{2} & -\frac{1}{\sqrt{20}} \\
\frac{1}{2} & -\frac{1}{\sqrt{20}} & -\frac{1}{2} & \frac{3}{\sqrt{20}} \\
\frac{1}{2} & \frac{1}{\sqrt{20}} & -\frac{1}{2} & -\frac{3}{\sqrt{20}} \\
\frac{1}{2} & \frac{3}{\sqrt{20}} & \frac{1}{2} & \frac{1}{\sqrt{20}}
\end{array}\right], \quad D_{3}=\left[\begin{array}{cccc}
\frac{1}{4} & 0 & 0 & 0 \\
0 & \frac{3}{20} & 0 & 0 \\
0 & 0 & \frac{1}{20} & 0 \\
0 & 0 & 0 & \frac{1}{140}
\end{array}\right] .
\end{aligned}
$$

\section{Degree elevation}

For raising the degree of Bézier curve by one without changing the shape of the curve. We can show that new vertices $c_{i}^{(1)}$ are obtained from the old polygon by piecewise linear interpolation at the parameter values $i /(n+1)$, see (Farin, 1993).

$$
c_{i}^{(1)}=\frac{i}{n+1} c_{i-1}+\left(1-\frac{i}{n+1}\right) c_{i}, \quad i=0,1, \ldots, n+1 .
$$

We can rewrite the formula (10) as a linear system $T_{n} c=c^{(1)}$, where the $(n+2) \times(n+1)$ matrix $T_{n}$ is

$$
T_{n}=\frac{1}{n+1}\left(\begin{array}{ccccccc}
n+1 & 0 & 0 & \ldots & 0 & 0 & 0 \\
1 & n & 0 & \ldots & 0 & 0 & 0 \\
0 & 2 & n-1 & \ldots & 0 & 0 & 0 \\
\vdots & \vdots & \vdots & \ddots & \vdots & \vdots & \vdots \\
0 & 0 & 0 & \ldots & n-1 & 2 & 0 \\
0 & 0 & 0 & \ldots & 0 & n & 1 \\
0 & 0 & 0 & \ldots & 0 & 0 & n+1
\end{array}\right)
$$


and the $(n+1)$ vector $c$ and the $(n+2)$ vector $c^{(1)}$ are

$$
\begin{aligned}
& c=\left(c_{0}, c_{1}, \ldots, c_{n}\right)^{\mathrm{t}}, \\
& c^{(1)}=\left(c_{0}^{(1)}, c_{1}^{(1)}, \ldots, c_{n}^{(1)}\right)^{\mathrm{t}} .
\end{aligned}
$$

We may repeat this process and then obtain a sequence of control points. After $r$ degree elevations, we have a linear system $T_{n, r} c=c^{(r)}$, where the $(n+r+1) \times(n+1)$ matrix

$$
T_{n, r}=T_{n+r-1} T_{n+r-2} \ldots T_{n+1} T_{n}
$$

has elements

$$
T_{n, r}(i, j)=\frac{\left(\begin{array}{c}
n \\
j
\end{array}\right)\left(\begin{array}{c}
r \\
i-j
\end{array}\right)}{\left(\begin{array}{c}
n+r \\
i
\end{array}\right)}, \quad i=0,1, \ldots, n+r \text { and } j=0,1, \ldots, n .
$$

By the orthogonality of Legendre basis, the degree elevation of a polynomial with Legendre basis is given by

$$
\begin{aligned}
& l=\left(l_{0}, l_{1}, \ldots, l_{n}\right)^{\mathrm{t}}, \\
& l^{(1)}=\left(l_{0}, l_{1}, \ldots, l_{n}, 0\right)^{\mathrm{t}} .
\end{aligned}
$$

After $r$ degree elevations, we have a linear system $\tilde{I}_{n, r} l=l^{(r)}$, where the $(n+r+1) \times(n+1)$ matrix $\tilde{I}_{n, r}$ has elements

$$
\tilde{I}_{n, r}(i, j)= \begin{cases}1 & \text { if } i=j, \\ 0 & \text { if } i \neq j .\end{cases}
$$

After transforming the Bernstein coefficients to the Legendre coefficients by $M_{n}^{-1}$, the degree elevation by $\tilde{I}_{n, r}$, and finding the Bernstein coefficients by $M_{n+r}$, we obtain the following theorem.

Theorem 6. The degree elevation matrix $T_{n, r}$ can be expressed in $M_{n}^{-1}, \tilde{I}_{n, r}$ and $M_{n+r}$ as

$$
T_{n, r}=M_{n+r} \tilde{I}_{n, r} M_{n}^{-1} .
$$

\section{Degree reduction}

When we find the best approximation in the sense of $L_{2}$-norm, in general, the degree reduction of Bézier curves address the following problem.

Problem 1 ( $L_{2}$ degree reduction). Let $\left\{c_{i}\right\}_{i=0}^{n}$ be a given set of control points which define the Bézier curve

$$
c^{n}(t)=\sum_{i=0}^{n} c_{i} B_{i}^{n}(t)
$$

of degree $n$. Then find another point set $\left\{b_{i}\right\}_{i=0}^{m}$ defining the approximative Bézier curve

$$
b^{m}(t)=\sum_{i=0}^{m} b_{i} B_{i}^{m}(t)
$$

of lower degree $m<n$ so that an $L_{2}$-distance function $d_{2}\left(b^{m}, c^{n}\right)$ between $b^{m}$ and $c^{n}$ is minimized. 
The $L_{2}$-distance of the two Bézier curves $b^{m}$ and $c^{n}$ is defined as following:

$$
d_{2}^{2}\left(b^{m}, c^{n}\right)=\int_{0}^{1}\left|b^{m}(t)-c^{n}(t)\right|^{2} \mathrm{~d} t=\int_{0}^{1}\left|\sum_{i=0}^{m} b_{i} B_{i}^{m}(t)-\sum_{i=0}^{n} c_{i} B_{i}^{n}(t)\right|^{2} \mathrm{~d} t .
$$

Using the matrix $T_{m, r}$, we can elevate the degree of $b^{m}$ from $m$ to $n$, where $r=n-m$,

$$
b^{(r)}=T_{m, r} b .
$$

Then the curve $b^{m}$ of degree $m$ is rewritten as a curve of degree $n$

$$
b^{m}(t)=b^{(r)}(t)=\sum_{i=0}^{n} b_{i}^{(r)} B_{i}^{n}(t),
$$

and the distance is

$$
d_{2}^{2}\left(b^{m}, c^{n}\right)=d_{2}^{2}\left(b^{(r)}, c^{n}\right)=\int_{0}^{1}\left|\sum_{i=0}^{n} b_{i}^{(r)} B_{i}^{n}(t)-\sum_{i=0}^{n} c_{i} B_{i}^{n}(t)\right|^{2} \mathrm{~d} t=\int_{0}^{1}\left|\sum_{i=0}^{n}\left(b_{i}^{(r)}-c_{i}\right) B_{i}^{n}(t)\right|^{2} \mathrm{~d} t .
$$

Thus we obtain the following theorem for the $L_{2}$-distance between the Bézier curve $b^{m}$ of degree $m$ and the Bézier curve $c^{n}$ of degree $n$.

Theorem 7. The $L_{2}$-distance between the two Bézier curves $b^{m}$ and $c^{n}$ is

$$
d_{2}^{2}\left(b^{m}, c^{n}\right)=d_{2}^{2}\left(b^{(r)}, c^{n}\right)=A^{\mathrm{t}} Q_{n} A,
$$

where $A=c-T_{m, r} b, b=\left(b_{0}, b_{1}, \ldots, b_{m}\right)^{\mathrm{t}}$ and $c=\left(c_{0}, c_{1}, \ldots, c_{n}\right)^{\mathrm{t}}$.

For developing the method, rewrite $d_{2}^{2}\left(b^{m}, c^{n}\right)$.

$$
\begin{aligned}
d_{2}^{2}\left(b^{m}, c^{n}\right) & =A^{\mathrm{t}} Q_{n} A \\
& =\left[c-T_{m, r} b\right]^{\mathrm{t}} Q_{n}\left[c-T_{m, r} b\right] \\
& =c^{\mathrm{t}} Q_{n} c-2 b^{\mathrm{t}} T_{m, r}^{\mathrm{t}} Q_{n} c+b^{\mathrm{t}} T_{m, r}^{\mathrm{t}} Q_{n} T_{m, r} b .
\end{aligned}
$$

One method of obtaining the vector $b$ is so-called the method of least squares (Lee and Park, 1997; Lutterkort et al., 1999). This method consists of minimizing $A^{\mathrm{t}} Q_{n} A$ with respect to $b$. We choose the vector $\hat{b}$ as that the value of $b$ minimizes $A^{\mathrm{t}} Q_{n} A$. Equating $\partial\left(A^{\mathrm{t}} Q_{n} A\right) / \partial b$ to zero and writing the resulting equations in terms of $\hat{b}$, we find that these equations are

$$
T_{m, r}^{\mathrm{t}} Q_{n} T_{m, r} \hat{b}=T_{m, r}^{\mathrm{t}} Q_{n} c .
$$

They are known as the normal equations.

Theorem 8. The $(n+1) \times(n+1)$ matrix $T_{n-1}^{\mathrm{t}} Q_{n} T_{n-1}$ has the following property:

$$
T_{n-1}^{\mathrm{t}} Q_{n} T_{n-1}=Q_{n-1} .
$$




\section{Proof.}

$$
\begin{aligned}
T_{n-1}^{\mathrm{t}} Q_{n} T_{n-1}(i, j) & =\frac{1}{2 n+1} \sum_{l=0}^{n+1} \frac{\left(\begin{array}{c}
n-1 \\
i
\end{array}\right)\left(\begin{array}{c}
1 \\
l-i
\end{array}\right)}{\left(\begin{array}{c}
n \\
l
\end{array}\right)} \sum_{k=0}^{n+1} \frac{\left(\begin{array}{c}
n \\
l
\end{array}\right)\left(\begin{array}{c}
n \\
k
\end{array}\right)}{\left(\begin{array}{c}
2 n \\
l+k
\end{array}\right)} \frac{\left(\begin{array}{c}
n-1 \\
j
\end{array}\right)\left(\begin{array}{c}
1 \\
k-j
\end{array}\right)}{\left(\begin{array}{c}
n \\
k
\end{array}\right)} \\
& =\frac{1}{2 n+1}\left(\begin{array}{c}
n-1 \\
i
\end{array}\right)\left(\begin{array}{c}
n-1 \\
j
\end{array}\right) \sum_{l=0}^{n+1} \sum_{k=0}^{n+1} \frac{\left(\begin{array}{c}
1 \\
l-i
\end{array}\right)\left(\begin{array}{c}
1 \\
k-j
\end{array}\right)}{\left(\begin{array}{c}
2 n \\
l+k
\end{array}\right)} \\
& =\frac{1}{2 n-1} \frac{\left(\begin{array}{c}
n-1 \\
i
\end{array}\right)\left(\begin{array}{c}
n-1 \\
j
\end{array}\right)}{\left(\begin{array}{c}
2 n-2 \\
i+j
\end{array}\right)}, \quad i, j=0,1, \ldots, n-1 .
\end{aligned}
$$

From Theorem 8, we have $T_{m, r}^{\mathrm{t}} Q_{n} T_{m, r}=Q_{m}$. Hence, the real symmetric positive definite matrix $T_{m, r}^{\mathrm{t}} Q_{n} T_{m, r}$ is invertible. Provided $\left(T_{m, r}^{\mathrm{t}} Q_{n} T_{m, r}\right)^{-1}$ exists, we have the unique solution for $\hat{b}$,

$$
\hat{b}=\left(T_{m, r}^{\mathrm{t}} Q_{n} T_{m, r}\right)^{-1} T_{m, r}^{\mathrm{t}} Q_{n} c .
$$

The approximate curve given by (11) is the best approximation with respect to the $L_{2}$-norm.

By the orthogonality of Legendre basis, the degree reduction of a polynomial with Legendre basis is given by

$$
\begin{aligned}
& l=\left(l_{0}, l_{1}, \ldots, l_{n}\right)^{\mathrm{t}}, \\
& l^{(-1)}=\left(l_{0}, l_{1}, \ldots, l_{n-1}\right)^{\mathrm{t}} .
\end{aligned}
$$

After $r$ degree reductions, we have a linear system

$$
\tilde{I}_{n,-r} l=l^{(-r)},
$$

where the $(n-r+1) \times(n+1)$ matrix $\tilde{I}_{n,-r}$ is

$$
\tilde{I}_{n,-r}=\left(\begin{array}{cccccccc}
1 & 0 & \ldots & 0 & 0 & 0 & \ldots & 0 \\
0 & 1 & \ldots & 0 & 0 & 0 & \ldots & 0 \\
\vdots & \vdots & \ddots & \vdots & \vdots & \vdots & \vdots & \vdots \\
0 & 0 & \ldots & 1 & 0 & 0 & \ldots & 0 \\
0 & 0 & \ldots & 0 & 1 & 0 & \ldots & 0
\end{array}\right) .
$$

After transforming the Bernstein coefficients to the Legendre coefficients by $M_{n}^{-1}$, the degree reduction by $\tilde{I}_{n,-r}$, and finding the Bernstein coefficients by $M_{m}$, we obtain the following theorem.

Theorem 9. The degree reduction matrix can be expressed in $M_{n}^{-1}, \tilde{I}_{n,-r}$ and $M_{m}$ as

$$
\left(T_{m, r}^{\mathrm{t}} Q_{n} T_{m, r}\right)^{-1} T_{m, r}^{\mathrm{t}} Q_{n}=M_{m} \tilde{I}_{n,-r} M_{n}^{-1}
$$

For the degree reduction with Bernstein basis, we can use the explicit matrix forms of $M_{m}, M_{n}^{-1}$ and $\tilde{I}_{n,-r}$ to compute $M_{m} \tilde{I}_{n,-r} M_{n}^{-1}$ given by the formula (4), (5) and (12), respectively. Therefore, our method using the relationship of transformations between Legendre and Bernstein basis is a simple and efficient method for optimal multiple degree reductions with respect to the $L_{2}$-norm. However, this best approximation does not in general interpolate the given curve at its endpoints. Thus we have to consider the smoothness of our method for the practical use. 
Here is the example given by the explicit matrix form and we have the same results with (Lutterkort et al., 1999).

Example 4 (Parametric case, $n=4)$.

$$
\begin{aligned}
& c=[0,1,2,1,0]^{\mathrm{t}}, \\
& l=M_{4}^{-1} c=\left[\frac{4}{5}, 0,-\frac{6 \sqrt{5}}{35}, 0, \frac{2}{105}\right]^{\mathrm{t}}, \\
& \left\|c^{4}\right\|_{2}^{2}=\left(\frac{4}{5}\right)^{2}+\left(\frac{6 \sqrt{5}}{35}\right)^{2}+\left(\frac{2}{105}\right)^{2}, \\
& M_{2} \tilde{I}_{4,-2} M_{4}^{-1}=\frac{1}{35}\left[\begin{array}{ccccc}
31 & 9 & -3 & -5 & 3 \\
-13 & 17 & 27 & 17 & -13 \\
3 & -5 & -3 & 9 & 31
\end{array}\right] \text {, } \\
& l^{(-2)}=\tilde{I}_{4,-2} M_{4}^{-1} c=\left[\frac{4}{5}, 0,-\frac{6 \sqrt{5}}{35}\right]^{\mathrm{t}}, \\
& c^{(-2)}=M_{2} \tilde{I}_{4,-2} M_{4}^{-1} c=\left[\frac{-2}{35}, \frac{88}{35}, \frac{-2}{35}\right]^{\mathrm{t}} \text {, } \\
& \left\|c^{(-2)}\right\|_{2}^{2}=\left(\frac{4}{5}\right)^{2}+\left(\frac{6 \sqrt{5}}{35}\right)^{2} \text {. }
\end{aligned}
$$

\section{Acknowledgements}

This work was supported by Dongseo University, Dongseo Frontier Project Research Fund of 2002 and Korea Research Foundation under grant KRF-99-015-DP0037.

\section{References}

Eck, M., 1993. Degree reduction of Bézier curves. Computer Aided Geometric Design 10, 237-251.

Eck, M., 1995. Least squares degree reduction of Bézier curves. Computer-Aided Design 27, 845-851.

Farin, G., 1993. Curves and Surfaces for Computer Aided Geometric Design, 3rd Edition. Academic Press, Boston.

Farouki, R.T., 2000. Legendre-Bernstein basis transformations. J. Comput. Appl. Math. 119, 145-160.

Lee, B.G., Park, Y., 1997. Distance for Bézier curves and degree reduction. Bull. Australian Math. Soc. 56, $507-515$.

Li, Y.M., Zhang, X.Y., 1998. Basis conversion among Bézier, Tchebyshev and Legendre. Computer Aided Geometric Design 15, 637-642.

Lutterkort, D., Peters, J., Reif, U., 1999. Polynomial degree reduction in the $L_{2}$-norm equals best Euclidean approximation of Bézier coefficients. Computer Aided Geometric Design 16, 607-612.

Lyche, T., Scherer, K., 2000. On the p-norm condition number of the multivariate triangular Bernstein basis. J. Comput. Appl. Math. 119, 259-273.

Mazure, M.-L., 1999. Chebyshev-Bernstein bases. Computer Aided Geometric Design 16, 649-669.

Watkins, M., Worsey, A., 1988. Degree reduction for Bézier curves. Computer-Aided Design 20, 398-405. 